\title{
TWO-BEAM INTERFERENCE OF LONG WAVELENGTH SYNCHROTRON RADIATION
}

\author{
G.L. Carr $^{\dagger}$ S.L. Kramer, BNL, Upton, NY \\ N. Jisrawi, Univ. of Jordan \\ L. Mihaly, and D. Talbayev, SUNY, Stony Brook, NY
}

\begin{abstract}
The synchrotron radiation emitted from the NSLS VUV ring is found to contain significant spectral structure at long wavelengths $(\lambda>0.5 \mathrm{~mm})$, suggesting multiple beam interference effects. A predominant pattern occurs as a result of direct radiation interfering with radiation reflected from the curved outer wall of the electron beam dipole chambers. This structure appears for both normal incoherent synchrotron radiation emission and coherent emission, the latter resulting from an instability-driven density modulation within the electron bunch.
\end{abstract}

\section{INTRODUCTION}

Long wavelength infrared (millimeter wave) synchrotron radiation is being developed at the NSLS to study the electronic properties of materials, but is also used to study longitudinal bunch instabilities that produce bursts of coherent synchrotron radiation[1]. The bursts have varying spectral content in the wavelength range from $2 \mathrm{~mm}$ to $>20 \mathrm{~mm}$, suggesting a high frequency contribution to the longitudinal impedance.

It has long been recognized that the conducting chamber of an electron storage ring will not sustain synchrotron radiation emission beyond a cutoff wavelength that obeys

$$
\lambda_{c} \cong 2\left(h^{3} / \rho\right)^{1 / 2}
$$

where $h$ is the vertical dimension (height) of the beam chamber, and $\rho$ is the orbit radius[2]. Since electron bunches are typically longer than this wavelength, multiparticle coherent synchrotron radiation is usually suppressed. But a short bunch, or a short wavelength density modulation within a long bunch, can radiate coherently. Under some circumstances, the radiation impedance for coherent emission may actually be sufficient to produce a density modulation within a long bunch[3], and the spectral character of synchrotron radiation near the beampipe cutoff wavelength may provide insight into the details of the radiation impedance.

Here we describe some of the spectral properties for

\footnotetext{
"Work supported by the U.S. Dept. of Energy through contract DE-AC02-98CH10886 at the NSLS.

† Email: carr@bnl.gov
}

long wavelength synchrotron radiation measured at the NSLS VUV ring using beamline U12IR. The spectra show large intensity oscillations ("fringes") for wavelengths longer than $0.5 \mathrm{~mm}$. This wavelength is longer than that typically achieved at other infrared beamlines, so these spectral fringes were not detected previously. This pattern is observed for both conventional (incoherent) and coherent synchrotron radiation emission. The shape of this fringe pattern is characteristic of twobeam interference, suggesting two effective source points. We have identified the extra source as an upstream tangent point, reflected from the outer wall of the dipole chamber. The fringe pattern is sensitive to horizontal electron beam motion of $\geq 10 \mu \mathrm{m}$.

\section{EXPERIMENT}

\subsection{NSLS VUV Ring}

The VUV ring at the National Synchrotron Light Source (NSLS/BNL) is an electron storage ring operated as a dedicated synchrotron radiation source. The bending radius is $1.91 \mathrm{~m}$ in each of the 8 dipole magnets. The ring chamber has a rectangular cross section, $4 \mathrm{~cm}$ high and $8 \mathrm{~cm}$ wide, except in some of the straight sections where it is circular. The orbit is $51 \mathrm{~m}$ in circumference and the 52.88 MHz RF system supports up to 9 electron bunches. Typical bunch lengths are about $30 \mathrm{~cm}(\sim 1 \mathrm{~ns})$. The cutoff wavelength (calculated using eq. 1) is $\lambda_{c} \cong 1.2 \mathrm{~cm}$ or $26 \mathrm{GHz}$.

\subsection{U12IR beamline and spectrometers}

The U12IR beamline is one of 6 infrared beamlines at the NSLS built to extract infrared synchrotron radiation from a bending magnet port[4]. The beamline extracts 90 $\mathrm{mr}$ by $90 \mathrm{mr}$ of radiation through a $6 \mathrm{~cm}$ wide aperture, and transports it (via mirror optics) through a diamond window to a Michelson interferometer (spectrometer) and $\ell$ He cooled bolometric detector. The diamond window transmits light across the entire IR spectral region, but its small diameter prevents wavelengths longer than $5 \mathrm{~mm}$ to be efficiently transmitted to the spectrometer.

The interferometer is capable of path differences greater than $1 \mathrm{~m}$ and spectral resolution better than $0.01 \mathrm{~cm}^{-1}$. The detected signal as a function of path difference (an "interferogram") is Fourier transformed to extract the spectral intensity. However, the interferogram itself often 
provides better insight when investigating the effects of multiple sources.

We extract even longer wavelengths through a large $(\sim 10 \mathrm{~cm}$ diameter) glass viewport situated upstream of the diamond window. This light is collected and detected with standard microwave components (waveguide horn, antennae, RF spectrum analyzers and diode detectors). The longest wavelengths detectable in this manner are greater than $10 \mathrm{~cm}(3 \mathrm{GHz})$.

\subsection{Spectral intensity}

The spectral intensity was measured using both conventional (incoherent) synchrotron radiation emission, and also bursts of coherent synchrotron radiation emission. The large coherent emission intensity was necessary for collecting spectra at microwave frequencies, as the RF detectors were not sufficiently sensitive to detect the small amount of incoherent light.

We measured the spectral intensity from the U12IR port using far infrared interferometer and a filter to limit the spectral content to below $20 \mathrm{~cm}^{-1}(=600 \mathrm{GHz})$. A typical spectrum. (see Fig. 1) shows a fringe pattern with a period of about $0.97 \mathrm{~cm}^{-1}(29 \mathrm{GHz})$. This pattern appears only for frequencies below about $20 \mathrm{~cm}^{-1}$, with the fringe contrast approaching almost $100 \%$ below $5 \mathrm{~cm}^{-1}$.

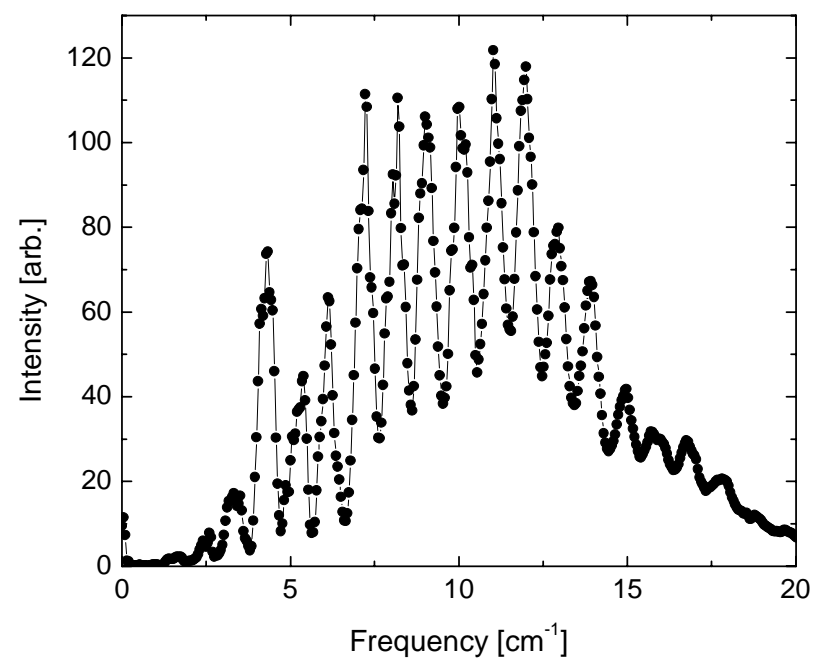

Figure 1: Far-infrared (mm wave) spectral intensity.

Further insight into the nature of this fringe pattern can be found from the far infrared interferogram, shown in Fig. 2. The main interference pattern around zero path difference (ZPD) contains information on the overall spectral content of the source and the spectral efficiency of the spectrometer and detector. A second interference pattern appears around $1 \mathrm{~cm}$ path difference, although inverted and smaller in magnitude. This behavior is consistent with a secondary source, coherent with the primary source, but situated about $1 \mathrm{~cm}$ closer or more distant. The inverted pattern is consistent with reflection from a metallic surface.

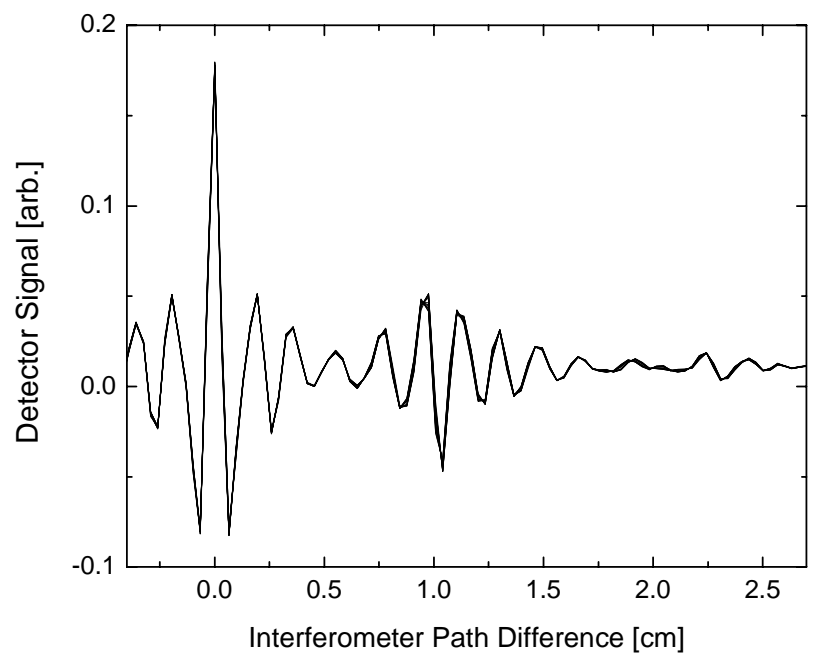

Figure 2: Interferogram signal at U12IR. The primary interference feature is centered at zero path difference. The smaller feature near $1.0 \mathrm{~cm}$ path difference is due to interference with a reflected beam.

We identified a plausible geometry inside the electron beam dipole chamber for a reflected source image. A schematic of the geometry is shown in Fig. 3. Consider a single electron traveling along the orbit shown as the dashed curve. The electron emits SR at all points along this arc. A beamport (e.g., U12IR) collects the SR from source tangent point $a$, but also from source point $c$ due to a reflection from the outer chamber wall at point $b$. The light travels long the line segments $c b$ and $b a$, whereas the electron travels along the radial arc from $c$ to $a$. The distances differ by about $1 \mathrm{~cm}$, i.e., the reflected SR experiences a phase lag of $1 \mathrm{~cm}$ plus a $\pi$ phase change due to reflection.

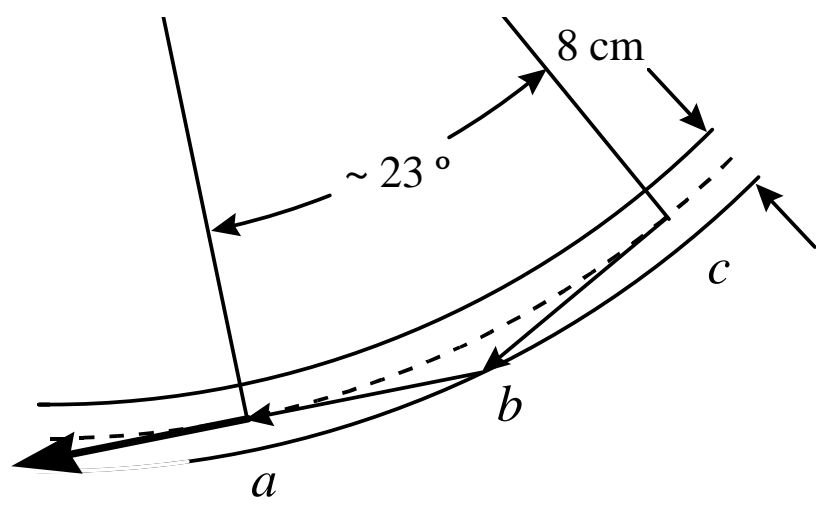

Fig. 3. Reflected light schematic, showing the electron trajectory (dashed line), source points $a$ and $c$, and reflection point $b$.

It is not surprising that the fringe contrast is greatest at the longest wavelength. In the far-field, the diffractionlimited apparent source size is $\sim 1.6\left(\lambda^{2} \rho\right)^{1 / 3}$. The size for $\lambda=3 \mathrm{~mm}\left(3 \mathrm{~cm}^{-1}\right)$ is $4 \mathrm{~cm}$, which is also the height of the beam chamber. Thus, both sources appear to emanate 
from same aperture (where the U12IR beamline begins) and overlap almost completely.

To test this hypothesis, we adjusted the orbit to produce a reasonably uniform horizontal shift of the beam in the relevant dipole and measured the resulting path difference using the interferometer, noting that path difference decreases as the orbit approaches the outer wall. The orbit shift (a total of $0.3 \mathrm{~mm}$ ) was measured using the beam position monitors (BPMs) immediately before and after this location and then averaging. The data is shown in Fig. 4 (solid circles) along with the calculated path difference (solid gray line). Agreement is very good. The measurement is sensitive enough to detect $10 \mu \mathrm{m}$ changes in the horizontal beam position relative to the outside wall.

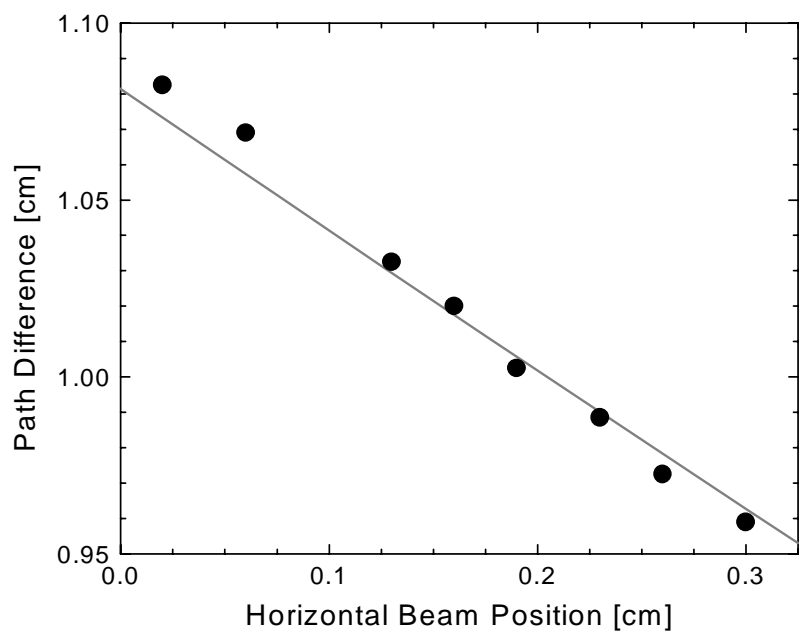

Figure 4: Measured (solid circles) and calculated (solid line) path difference for the chamber wall reflection.

This interference fringe persists to lower frequencies, as detected in the coherent emission spectrum. This is shown in Fig 5 for both the far-infrared and microwave spectral ranges. We do not have absolute measurements of the intensity, so the level for the far-infrared was scaled to match the microwave data for comparison. Surprisingly, significant intensity is detected down to $\sim 6 \mathrm{GHz}$, a factor of four beyond the cutoff wavelength. This emission is found to correlate with streak camera data, showing a large density modulation with a $5 \mathrm{~cm}$ wavelength[5]. We note that the fringe pattern changes at these long wavelengths, perhaps due to a second type of reflection where synchrotron radiation intercepts the top and bottom surfaces of the ring chamber, and reflection from the chamber corner occurs. The path difference is only slight (about $10 \%$ longer), but the light experiences a $2 \pi$ phase change, resulting in a maximum toward zero frequency. Beating between these two patterns may account for the complex behavior near $6 \mathrm{~cm}^{-1}$ (see Fig. 1).

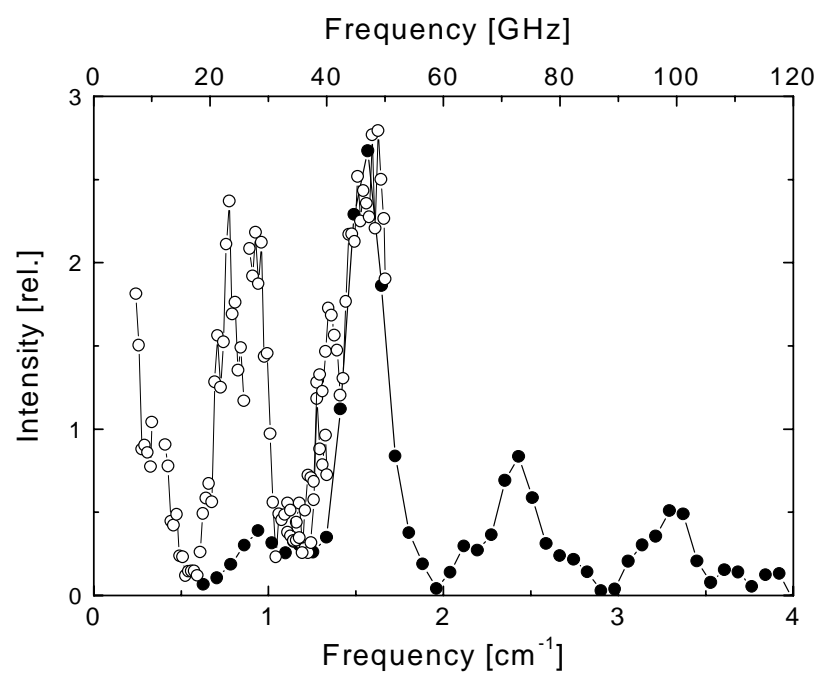

Figure 5: Coherent emission spectral intensity for both far infrared and RF detectors.

\section{SUMMARY}

The millimeter wave spectra for the NSLS VUV ring contains complex structure, some of which has been identified as multiple source reflection inside the ring chamber. It is noteworthy that we observe bursts of coherent emission in this same spectral range. These bursts appear to result from a longitudinal density modulation, most likely induced by the microwave instability where electrons in a bunch interact through their wakefield. The impedance that characterizes this interaction has a number of sources, including the radiation impedance from synchrotron radiation emission. The complex spectra suggest this impedance may have strong resonant characteristic that could influence the instability and contribute to this coherent emission. Further studies are planned to investigate this possibility and the potential for $\mathrm{CW}$ excitation of this coherent radiation power.

\section{ACKNOWLEDGEMENTS}

We are grateful for assistance from the NSLS operations staff and useful discussions with J.B. Murphy, N. Towne and B. Podobedov.

\section{REFERENCES}

[1] G.L. Carr et al., Nucl. Instrum. Meth. Sec A.463, 387 (2001).

[2] R.L. Warnock, SLAC-PUB-5375 (1990).

[3] S. Heifets and G. Stupakov, SLAC-PUB-8761 (2001) and these Proceedings.

[4] R.P.S.M. Lobo et al, Rev. Sci. Instrum. 96, 180 (1999).

[5] B. Podobedov et al., these Proceedings. 